\title{
CERATOCONJUNTIVITE SECA EM CÃES
}

Giovana Alves Leandro1, Luiz Antonio de Jesus Sousa', Rayanne Borges Vieira1, Wanessa Ferreira Ataíde ${ }^{1}$, Andréia Vitor Couto do Amaral²

1- Médico(a) Veterinário(a) Residente em Clínica, Cirurgia e Anestesiologia de Pequenos Animais, Universidade Federal de Jataí, Jataí - GO, Brasil. E-mail: luizantoniomukies@hotmail.com

2- Professora doutora do Programa de Residência Multiprofissional em Saúde, Universidade Federal de Jataí, Jataí - GO, Brasil.

Recebido em: 22/09/2018 - Aprovado em: 23/11/2018 - Publicado em: 03/12/2018 DOI: 10.18677/EnciBio 2018B119

\begin{abstract}
RESUMO
A Ceratoconjuntivite seca (CCS) é uma doença oftálmica comum em cães, em que é observada a deficiência do filme lacrimal pré-corneal. Pode ser classificada como quantitativa ou qualitativa. A CCS quantitativa cursa com a diminuição do componente aquoso do filme lacrimal, reconhecidamente a mais comum na rotina clínica. A CCS qualitativa cursa com a diminuição do componente lipídico ou mucoso do filme lacrimal. Clinicamente os cães com CCS apresentam processo inflamatório crônico da superfície corneal na maioria dos casos, manifestada com opacidade, neovascularização corneal, secreção seromuscular, hiperemia conjuntival e pigmentação corneal. O diagnóstico se dá por meio da utilização do teste lacrimal de Schirmer para a CCS quantitativa, sendo considerado suspeitos animais que apresentem valores menores que $15 \mathrm{~mm} / \mathrm{min}$ e confirmatório animais que apresentem valores menores que $10 \mathrm{~mm} / \mathrm{min}$, e por meio do teste do tempo de ruptura do filme lacrimal para a CCS qualitativa, podendo ainda ser empregado a rosa bengala e teste de osmolaridade lacrimal. São várias as causas de CCS, sendo a imunomediada a principal. O tratamento terapêutico é mais indicado, sendo que a escolha do fármaco irá depender da causa da CCS. Os principais fármacos utilizados são os imunomoduladores para a CCS qualitativa de origem imunomediada e os lacrimomiméticos ou substitutos da lágrima para a CCS qualitativa. Os tratamentos cirúrgicos e novos tratamentos podem ser empregados mediante o insucesso dos tratamentos convencionais. Diante da elevada incidência de cães acometidos com ceratoconjuntivite seca, o objetivo do trabalho foi discorrer sobre as principais causas dessa doença, os sinais clínicos, as formas de diagnóstico e as opções disponíveis para tratamento.
\end{abstract}

PALAVRAS-CHAVE: Filme lacrimal, imunomediadas, opacidade corneana, teste de Schirmer

\section{KERATOCONJUNCTIVITIS SICCA IN DOGS - LITERATURE REVIEW}

\section{ABSTRACT}

Dry keratoconjunctivitis (CCS) is a common ophthalmic disease in dogs, caused by deficiency of the precorneal tear film. It can be classified as quantitative or qualitative. Quantitative CCS is associated with a decrease in the aqueous 
component of the tear film, which is recognized as the most common clinical routine. The qualitative CCS courses with the decrease of the lipid or mucous component of the tear film. Clinically, dogs with CCS present a chronic inflammatory process of the corneal surface in most cases, manifested with opacity, corneal neovascularization, seromuscular secretion, conjunctival hyperemia and corneal pigmentation. The diagnosis is made through the use of the Schirmer lacrimal test for quantitative SCC, where in dogs values below $15 \mathrm{~mm} / \mathrm{min}$ are suspected and less than $10 \mathrm{~mm} / \mathrm{min}$ confirms the diagnosis, and by means of the rupture time test of the lacrimal film for the qualitative CCS, and pink cane and lacrimal osmolarity test may be used. There are several causes of CCS, with immunomediation being the main cause. Therapeutic treatment is mainly indicated, and the choice of drug will depend on the cause of CCS. The main drugs used are the immunomodulators for qualitative CCS of immunomediated origin and the lachryomimetics or tear substitutes for qualitative CCS. Surgical treatments and new treatments can be employed through the failure of conventional

treatments. In view of the high incidence of dogs with dry keratoconjunctivitis, the objective of the study was to discuss the main causes of this disease, the clinical signs, the forms of diagnosis and the options available for treatment.

KEYWORDS: Lacrimal film, immunomediated, corneal opacity, Schirmer test

\section{INTRODUÇÃO}

A Ceratoconjuntivite seca (CCS), também conhecida como síndrome do olho seco, é uma das mais comuns oftalmopatias encontradas na rotina clínica de pequenos animais, e se caracteriza pela deficiência do filme pré-corneal. Tal deficiência poderá ser classificada como quantitativa ou qualitativa, ou por uma combinação de ambas, causando alterações importantes na superfície corneal (MOTTA et al., 2014). A CCS quantitativa é caracterizada pela diminuição da porção aquosa do filme lacrimal, já a CCS qualitativa se caracteriza pela alteração nos componentes mucosos e lipídicos do filme lacrimal (TILLEY; SMITH JUNIOR, 2008; COLITZ, 2008).

A predisposição racial da CCS é descrita na literatura, sendo os cães das raças Bulldog inglês, Pug, Yorkshire Terrier, Lhasa Apso, Shih-tzu, Cocker, Schnauzer miniatura, entres outras, notamente em cães braquicefálicos (HERRERA, 2008; BIONDI et al, 2010).

A CCS em cães possui várias origens etiológicas, dentre as quais, traumas orbital ou supraorbital, iatrogênica quando há remoção da glândula da terceira pálpebra ou da glândula lacrimal principal, por doenças infecciosas como cinomose canina, induzida por fármacos como sulfas e as imunomediadas, as quais representam a maior parte dos casos (COLITZ, 2008; CHERRY et al., 2018).

Os sinais clínicos variam conforme o tempo de surgimento da doença (agudo ou crônico), como também o grau e extensão de ressecamento ocular. Na maioria dos casos, o aparecimento é gradual e bilateral, aumentando conforme o tempo, sendo prejudicial a visão do animal e causando diversos sinais clínicos. Dentre os principais sinais clínicos comumente encontrados, o paciente pode apresentar hiperemia conjuntival, secreção seromucosa fortemente aderida à córnea e às pálpebras, dermatite periocular, blefaroespasmos, ressecamento ocular, edema e neovascularização corneal. Enquanto que a ulceração corneal é mais comum em casos agudos, a fibrose, a perda da transparência corneana com pigmentação e a diminuição ou perda da acuidade visual são alterações mais observadas em casos crônicos (ASTRAUKAS, 2013; MOTTA et al., 2014; JORGE 2015). 
O tratamento varia conforme a causa, a apresentação clínica, o grau de evolução da CCS e as alterações observadas nos exames específicos. O tratamento terapêutico constitui a primeira alternativa na maioria absoluta dos casos. Os principais fármacos utilizados são os imunomoduladores para a CCS qualitativa de origem imunomediada e os lacrimomiméticos ou substitutos da lágrima para a CCS qualitativa. Os tratamentos cirúrgicos e novos tratamentos podem ser empregados mediante o insucesso dos tratamentos convencionais (ASTRAUSKAS; CAMARGO, 2013; VOITENA, 2018).

Este trabalho de revisão de literatura tem por objetivo abordar as principais características da ceratoconjuntivite seca em cães, como também detalhar os principais sinais clínicos, os exames necessários para o diagnóstico e as opções de tratamento.

\section{Definição}

A Ceratoconjuntivite seca (CCS) é uma enfermidade ocular caracterizada por diminuição de um ou mais componentes do filme lacrimal e cursa com quadro clínico inflamatório na córnea e conjuntivas oculares, a depender da causa e do tempo de evolução da doença. São atribuídas várias etiologias à CCS, as quais promovem deficiências quantitativas ou qualitativas, ou ambas, do filme lacrimal précorneal, sendo a deficiência quantitativa observada na maioria dos casos (ASTRAUKAS; CAMARGO, 2013; FERRAZ; SOUZA, 2014).

\section{Fisiologia do filme lacrimal}

O sistema lacrimal é constituído pelos seguintes componentes: glândula lacrimal principal da órbita, glândula da terceira pálpebra, glândulas lacrimais acessórias, glândulas de Meibômio, células caliciformes da conjuntiva, filme lacrimal pré-corneano, filamentos mucosos, pontos e canalículos lacrimais, ducto nasolacrimal, ponto nasal e saco nasolacrimal. Os componentes secretores do sistema lacrimal incluem as glândulas lacrimais acessórias e principal, a glândula da terceira pálpebra, as glândulas de Meibômio e as células caliciformes da conjuntiva (COLITZ, 2008; CASTILLO, 2012).

O filme lacrimal é uma camada muito importante entre a pálpebra e a superfície ocular e entre o meio externo e a superfície ocular. É predominantemente aquoso e é formado a partir das secreções das glândulas lacrimais, das glândulas de Meibômio e das células caliciformes conjuntivais (CASTILLO, 2012).

Em um passado recente, considerava-se que o filme lacrimal possuia três camadas bem distintas: uma superficial composta por lipídios, uma camada média aquosa e uma última camada composta por substâncias mucosas. Atualmente, sabe-se que que os compostos mucosos também são liberados na camada aquosa, enquanto que algumas mucinas estão fortemente ligadas ao epitélio corneal (WILLIAMS, 2017).

Os compostos lipídicos, que mantem-se na superfície do filme lacrimal, evitam a evaporação da lágrima. São produzidos pelas glândulas de Meibômio, que constituem são glândulas sebáceas adaptadas que se localizam na borda palpebral. O produto secretado é um material proteico, fosfolipídico, modulado por hormônios, tais como andrógenos, progestogênio, estrogênio, hormônio liberador de corticotrofina e substância $\mathrm{P}$, liberado na superfície ocular durante os movimentos palpebrais. Durante o sono e em períodos em que os movimentos palpebrais estão reduzidos, este produto tende a se acumular nos ductos das glândulas meibomianas, podendo ser liberado em maior quantidade. (CASTILLO, 2012). A 
deficiência destes compostos lipídicos da lágrima acarreta em maior evaporação da lágrima, aumento da sua osmolaridade e irritação ocular (JORGE et al., 2015).

Os compostos aquosos são os maiores constituintes, em termos de quantidade, do filme lacrimal pré-corneal. Por essa razão, a sua deficiência resulta na CCS quantitativa. Os compostos aquosos são produzidos pela secreção da glândula lacrimal principal, da glândula lacrimal da terceira pálpebra e de pequenas glândulas lacrimais acessórias. Esta secreção é consituída principalmente por eletrólitos, uréia, água, glicose, glicoproteínas e proteínas lacrimais. Suas principais funções constituem a nutrição, hidratação e proteção da córnea (BIONDI et al., 2012).

Os compostos mucosos são produzidos pelas células caliciformes distribuídas nas conjuntivas oculares. Estas células estão em maior concentração no fórnix conjuntival. O muco é importante para a fixação do filme lacrimal à superfície ocular, onde as mucinas irão ligar-se ao glicocálix nas microvilosidades das células do epitélio corneal. A mucina também dá viscosidade e volume ao filme lacrimal, dispergindo-se nos compostos aquosos (COLITZ, 2008; CASTILLO, 2012; WILLIAMS, 2017).

A lágrima também possui imunoglobulinas, lisozimas e outros mecanismos de defesa. Um deles é a lactoferrina, com ação anti-bacteriana, capaz de ligar ao ferro. As bactérias necessitam do ferro como componente fundamental na transferência de elétrons, e mediante uma potencial infecção, a conjuntiva ocular pode responder produzindo lactoferrina para se ligar ao máximo possível de ferro na lágrima (COLITZ, 2008; CASTILLO, 2012; WILLIAMS, 2017).

\section{Tipos de Ceratoconjuntivite Seca}

\section{Ceratoconjuntivite seca quantitativa}

A Ceratoconjuntivite seca quantitativa é caracterizada pela deficiência do componente aquoso do filme lacrimal pré-corneano, resultando em ressecamento e inflamação da conjuntiva e da córnea e dor ocular, entretanto os sinais clínicos irão depender na origem etiológica da CCS, da gravidade do quadro e também da fase em que se encontra a doença, ou seja, aguda ou crônica (COLITZ, 2008). É importante entender que os pacientes com CCS quantitativa também terão uma deficiência qualitativa, pois a fração aquosa da lágrima é necessária para estimular o deslizamento palpebral e a secreção de lipídios, além de, que sem esses compostos aquosos, a nutrição e produção das células caliciformes e das glândulas de Meibômio se alteram, prejudicando seus produtos (WILLIAMS, 2017; CHERRY et al., 2018).

\section{Ceratoconjuntivite seca qualitativa}

A Ceratoconjuntivite seca qualitativa se caracteriza pela alteração nos componentes lipídicos ou mucosos da lágrima. A deficiência de mucina causa instabilidade do filme lacrimal que resulta em ressecamento da córnea. Causas específicas de deficiência de mucina são doenças infecciosas ou imunomediadas, resultando em destruição de células caliciformes. Outras causas incluem cicatriz grave, após doença ulcerativa da conjuntiva, e deficiência de vitamina $A$, que provoca metaplasia escamosa da conjuntiva e subsequente perda de células caliciformes (TILLEY; SMITH JUNIOR, 2008). Os compostos lipídicos podem apresentar-se deficientes em processos inflamatórios das glândulas de Meibômio 
específicos ou que podem acompanhar as blefarites ou ainda relacionadas a alterações imunomediadas e idiopáticas (CHERRY et al., 2018).

\section{Causas de Ceratoconjuntivite seca em cães}

A CCS em cães possui diversas etiologias, porém acredita-se que a causa imunomediada seja a de maior prevalência. A adenite da imunomediada das glândulas lacrimais pode ocorrer associada a outras doenças autoimunes como a atopia, o hiperadrenocorticismo, o hipotireoidismo e a diabete (TILLEY; SMITH JUNIOR, 2008; CHERRY et al., 2018). Outras etiologias incluem as causas infecciosas, tais como a cinomose canina, a erliquiose, a toxoplasmose, a leptospirose e a leishmaniose; a causa iatrogênica como a exérese da glândula da terceira pálpebra; causas traumáticas como o trauma orbital ou supra-orbital; além de causas neurogênicas, que envolvam o nervo facial ou o nervo trigêmeo; causas congênitas, neoplásicas, tóxicas, metabólicas e radioterapia para neoplasias localizadas na cabeça já foram relacionadas com a CCS (PEIXOTO, 2013; ASTRAUKAS; CAMARGO, 2013; BARACHETTI et al., 2015; CHERRY et al., 2018).

As raças de cães mais acometidas pela ceratoconjuntivite seca são: Cocker Spaniel, Buldogue Inglês, Pugs, Yorkshire Terriers, Pequinês, English Springer Spaniel, West Highland, White Terriers, Shih-tzus e Boston Terriers. Foi observado que as fêmeas são mais acometidas do que os machos, bem como cães de raças braquicefálicas (HERRERA, 2008; BIONDI et al., 2010; PEIXOTO, 2013).

Sabe-se que na CCS imunomediada há infiltração de células T e B. A regulação positiva dessas moléculas promove a apresentação do antígeno, bem como o aumento da secreção de citocinas pró-inflamatórias, como o interferon (DODI, 2015). Este processo geralmente resulta em secreção ocular mucopurulenta, hiperemia de conjuntiva, ceratite, pigmentação corneana, e blefarospasmo em CCS (DESTEFANIS, 2016). Estudos histopatológicos realizados a partir de tecidos lacrimais de cães afetados com CCS idiopática revelaram graus variados de infiltrado celular linfocítico-plasmocitário, sugerindo assim um quadro de imunológico para a doença (BARACHETTI et al., 2015).

A resposta clínica aos imunomoduladores como ciclosporina, pimecrolimus e tacrolimus pode confirmar uma possível causa imunomediada, estabelecendo-se um diagnóstico terapêutico (DODI, 2015). Entretanto, deve-se levar em consideração que uma grande variedade de doenças autoimunes podem estar associadas os pacientes com CCS, devendo ao clínico esta investigação, dentre as quais o hipotireoidismo, diabetes melito, polimiosite, poliartrite, piodermatites, glomerulonefrite e a colite ulcerativa (SGRIGNOLI et al; 2013).

As causas iatrogênicas, como a exérese da glândula da terceira pálpebra, frequentemente levam a CCS em raças predispostas. Assim, o procedimento é terminantemente contraindicado em casos de prolapso da glândula da terceira pálpebra, qualquer que seja a raça ou espécie, mas, em especial, aqueles de raça citadas como predispostos ao CCS. É valido ressaltar que algumas raças são predispostas tanto à CCS quanto ao prolapso da glândula da terceira pálpebra, notadamente os braquicefálicos (BIRCHARD; SHERDING, 2008; TILLEY; SMITH JUNIOR, 2008; BIONDI, 2015).

Anestésicos locais ou sistêmicos, sedativos e a atropina podem causar temporariamente a CCS. Se o uso for interrompido, haverá um retorno geral para condições normais (DODI, 2015). Fármacos como, fenazopiridina, sulfadiazina, sulfametazol e sulfassalazina também podem induzir CCS em cães (ORIÁ, 2010). 
A CCS neurogênica poderá ser causada por trauma ou processos neoplásicos ou inflamatórios na região supra-orbital, temporal ou, mais raramente, na orelha interna, que possam acometer ramos do nervo trigêmeo ou do nervo facial. O nervo trigêmeo é responsável pela via aferente parassimpática de produção da lágrima, enquanto que o facial constitui a via eferente (LEIVA; GIMÉNEZ, 2017).

\section{Sinais clínicos}

Os sinais clínicos variam conforme o tempo de surgimento da doença como também o grau e extensão de ressecamento ocular. É uma doença que pode se apresentar de forma bilateral ou unilateral, crônica ou aguda e permanente ou temporária, podendo apresentar histórico inespecífico e recorrente. Na maioria dos casos, o imunomediado, o aparecimento é gradual e bilateral, aumentando conforme o tempo, sendo prejudicial a visão do animal e causando diversos sinais clínicos (ASTRAUKAS; CAMARGO, 2013; MOTTA et al., 2014; JORGE, 2015). Os principais sinais clínicos, dependendo da fase da CCS em que o cão se encontra, estão demonstrados no Quadro 1.

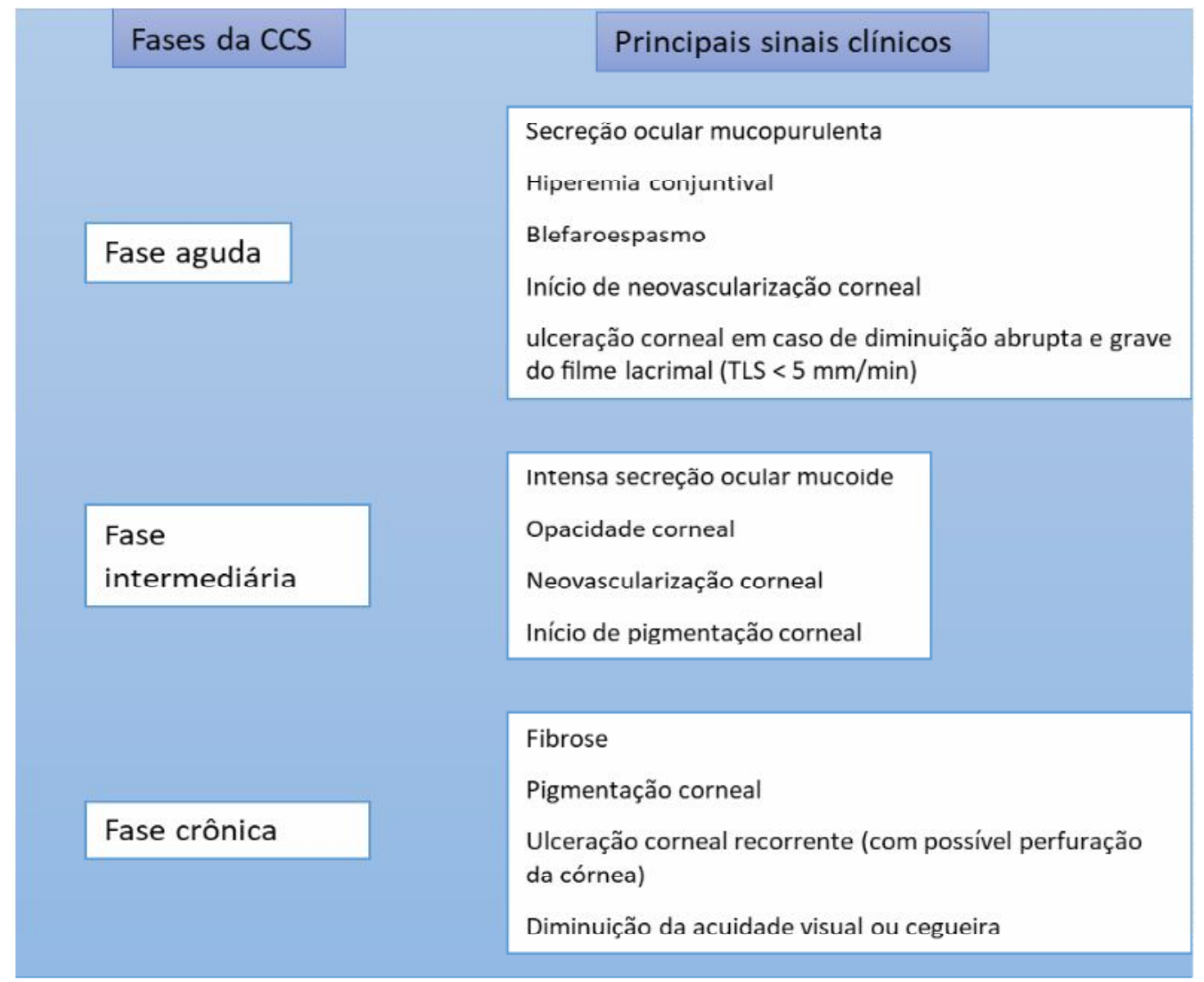

QUADRO 1 - Sinais clínicos mais frequentes na CCS de acordo com a fase em que a doença se encontra. Fonte: Adaptada de DODI (2015).

Quadros tóxicos e infecciosos frequentemente apresentam-se de maneira aguda, cursando, inclusive, com úlceras de córnea, devido ao ressecamento abrupto da superfície ocular. Já os quadros neurológicos são unilaterais e a narina ipslateral do paciente apresenta-se também ressecada, por receber ramificações dos mesmos nervos acometidos (LEIVA; GIMÉNEZ, 2017 ; WILLIAMS, 2017).

Inicialmente os olhos se apresentam hiperêmicos e inflamados, com secreção mucoide ou mucopurolenta intermitente. Dentre os principais sinais clínicos ENCICLOPÉDIA BIOSFERA, Centro Científico Conhecer - Goiânia, v.15 n.28; p. 3022018 
comumente encontrados o animal pode apresentar, sensibilidade ocular, dermatite periocular, blefaroespasmos, ressecamento, edema, quemose, neovascularização e ulceração corneal, além de fibrose e pigmentação ocular, sendo que nos casos mais crônicos o animal pode vir a apresentar, perda da transparência corneana e diminuição ou perda da acuidade visual (GELLAT, 2013; JORGE, 2015).

Nos casos de CCS aguda o olho pode apresentar acúmulo de secreção mucopurulenta, falta de brilho na córnea, blefaroespasmo e até mesmo úlceras de córnea dependendo da gravidade da diminuição quantitativa do filme lacrimal. Caso não seja instituído o tratamento imediato, poderá ocorrer agravamento do quadro e evolução para o estágio crônico, onde o animal vai apresentar espessamento, pigmentação e neovascularização da córnea, intensificando o desconforto sofrido e podendo levar a cegueira. $O$ acúmulo de secreções nas margens palpebrais e na pele periocular poderá provocar blefarite e dermatite cursando com alopecia periocular de graus variados (MOTTA et al., 2014; JORGE, 2015).

Com a progressão e cronicidade da doença, os sinais clínicos se intensificam podendo levar a blefaroespasmos recorrentes. Na CCS crônica há uma alteração em todo epitélio corneano que se torna hiperplásico com queratinização e presença de depósitos de melanina em todo o epitélio e estroma anterior, além de intensa vascularização, com isso o dano persistente na superfície ocular acaba progredindo para cegueira (HERRERA, 2008a; GELLAT, 2013). Alguns sinais clínicos estão demonstrados nas figuras 1 a 3.

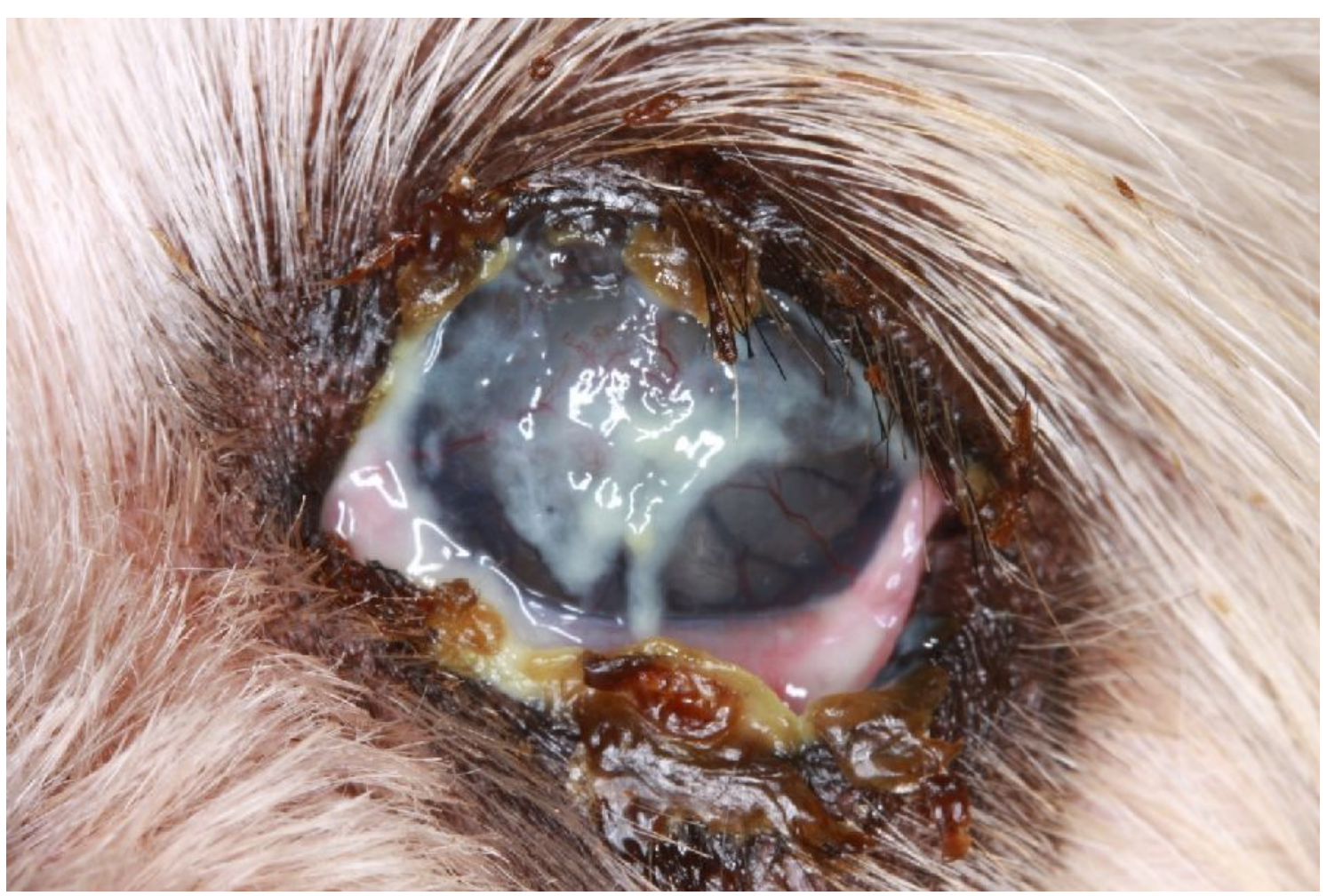

FIGURA 1 - Cão com CCS, apresentando secreção mucosa aderida à córnea e às pálpebras, neovascularização corneal e hiperemia conjuntival. Fonte: Fernando Bretas. 


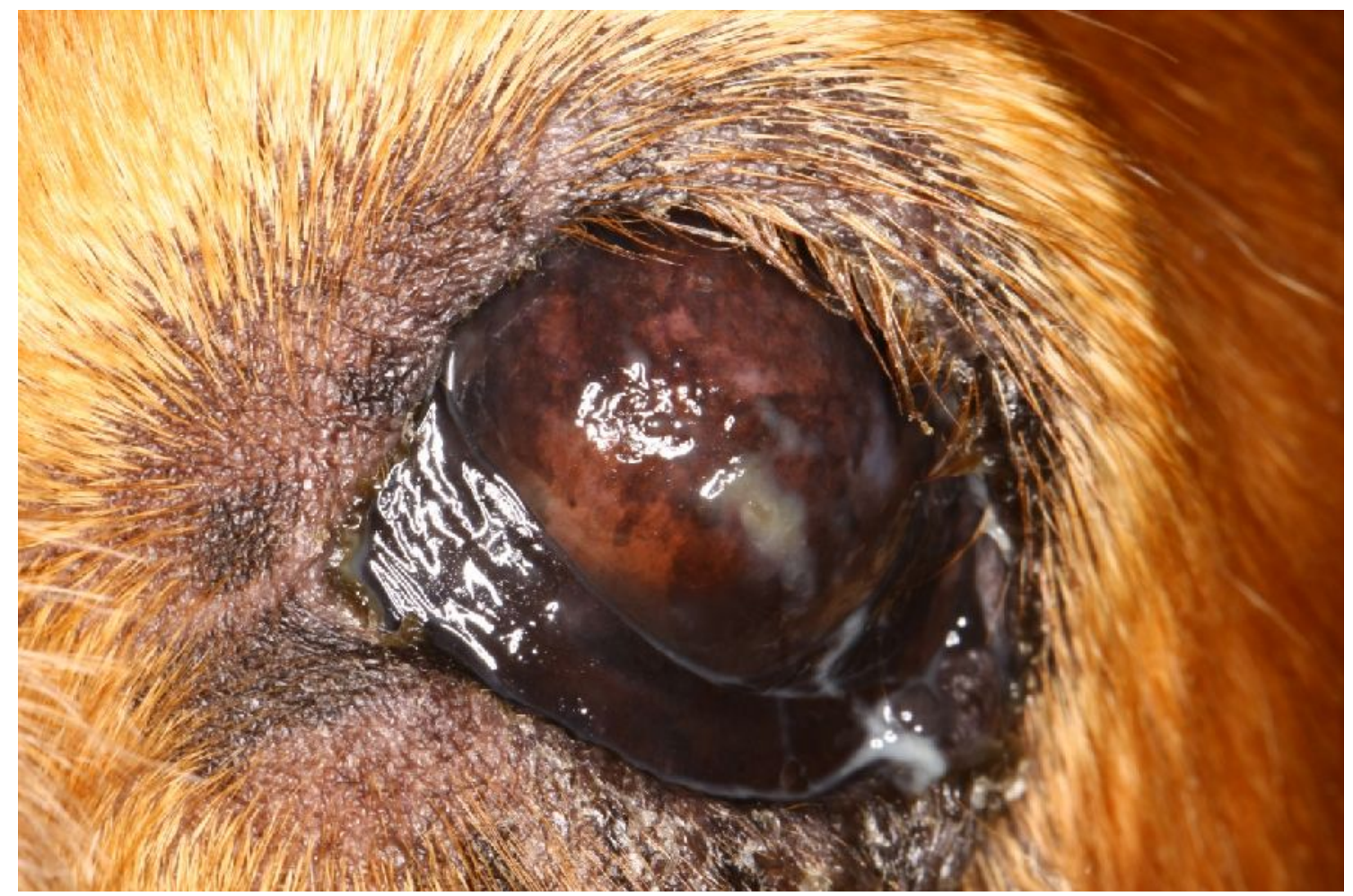

FIGURA 2 - Cão com CCS, apresentando secreção mucosa aderida à córnea e intensa pigmentação corneal. Fonte: Fernando Bretas.

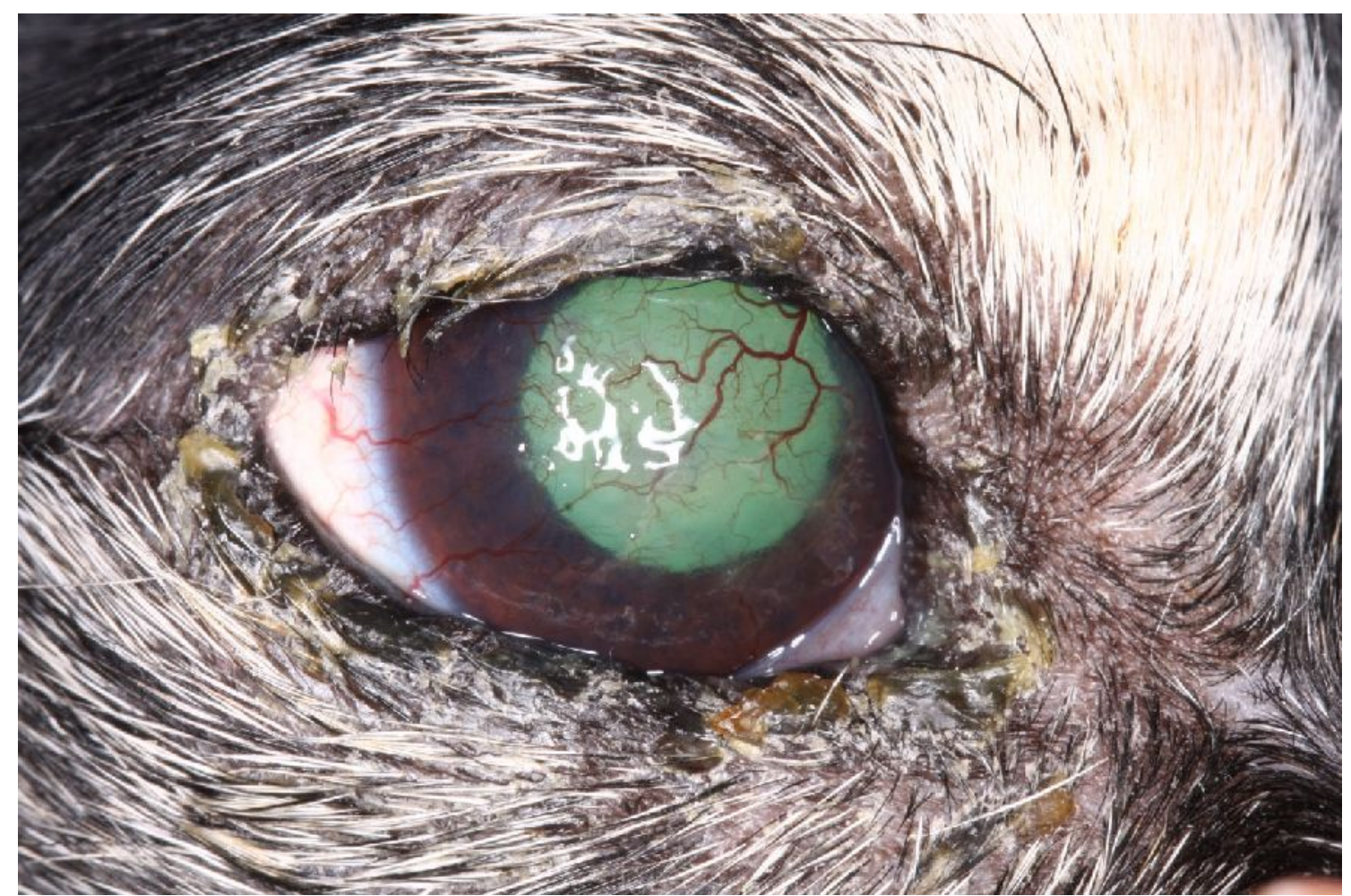

FIGURA 3 - Cão com CCS, apresentando secreção mucosa aderida às pálpebras, acentuada neovascularização corneal e discreto edema corneal. Fonte: Fernando Bretas.

Sintomas das alterações das glândulas de Meibômio podem ser variáveis. Meibomianite aguda e crônica pode provocar tumefação das margens palpebrais, com glândulas de Meibômio tamponadas. Podem ocorrer lesões em glândulas 
isoladas ou em várias glândulas, cursando com inclusive com blefarite, comumente de origem infecciosa ou associada com doenças dermatológicas, notadamente àquelas de natureza imunomediada (BIRCHARD ; SHERDING, 2008; LAUS, 2009).

\section{Diagnóstico}

O diagnóstico definitivo da Ceratoconjuntivite seca é baseado principalmente nos sinais clínicos apresentados pelo animal e nos resultados dos exames específicos. Como exames específicos, pode-se utilizar o corante Rosa Bengala, o teste de Fluoresceína, o teste do tempo de ruptura do filme lacrimal, teste de Schirmer (TLS) I e II e citologia conjuntival e esfoliativa (ANGELICO et al., 2011; ASTRAUKAS, 2013; MOTTA et al., 2014).

O teste de Rosa Bengala é considerado o teste padrão para avaliação da superfície conjuntival, corando áreas de descontinuidade do filme lacrimal, constituindo um bom método para auxiliar na detecção da CCS qualitativa. Com sua utilização é possível avaliar o grau de desvitalização das células epiteliais, defeitos agudos do epitélio tanto da córnea como da conjuntiva bulbar e filamentos de muco que possam estar aderidos (SLATTER, 2005; JORGE, 2015). O teste de Fluoresceina é o mais comumente utilizado na prática veterinária, sendo empregado para o diagnóstico de defeitos epiteliais da córnea e conjuntiva, visando detectar descontinuidade do epitélio corneano (SLATTER, 2005; COLITZ 2008; ASTRAUKAS; CAMARGO, 2013).

O teste de ruptura do filme lacrimal é um teste utilizado no diagnóstico de anormalidade qualitativa do filme lacrimal pré corneal (FLPC), consiste na aplicação tópica de fluoresceína 0,5\% e manter as pálpebras do paciente abertas, o tempo é contado a partir do último piscar até o aparecimento do primeiro ponto seco que se forma na córnea, a observação é realizada com auxílio de um biomicroscópio com lâmpada de fenda e filtro azul de cobalto. O tempo adequado para que haja a ruptura do filme lacrimal é de 20 segundo ou mais, ocorrendo em menos de 5 segundos em casos onde o paciente apresenta deficiência na produção de mucina lacrimal (COLITZ, 2008; JORGE, 2015).

O teste da lágrima de Schirmer (TLS) I e II é o teste padrão para avaliar a produção aquosa do filme lacrimal pré-corneal, sendo um método semiquantitativo de mensuração. $O$ teste pode ser realizado com ou sem utilização de anestesia tópica sendo mais recomendado sem anestesia tópica e bilateral quando for a primeira visita do animal. O método TLS-I mensura a produção basal e reflexa do filme lacrimal, incluindo a lágrima reflexa da estimulação corneal e do nervo trigêmeo que é realizada no momento da realização do teste (SLATTER, 2005; MOTTA et al., 2014; DODI, 2015). O teste consiste na utilização de tiras estéreis de papel absorvente milímetrado que são inseridos dentro do fórnix conjuntival palpebral inferior (Figura 4), onde deve permanecer por 1 minuto (SLATTER, 2005; DODI, 2015). O resultado deve ser interpretado imediatamente após a retirada da tira, sendo o resultado avaliado em milímetros por minuto $(\mathrm{mm} / \mathrm{min})$, mediante mensuração da área umedecida pela lágrima (MOTTA et al., 2014; DODI, 2015). A produção padrão de lágrimas em cães é acima de $15 \mathrm{~mm} / \mathrm{min}$, valores entre 10 e 15 $\mathrm{mm} / \mathrm{min}$ são sugestivos de CCS e resultados abaixo de $10 \mathrm{~mm} / \mathrm{min}$ são considerados positivos, principalmente quando apresentam sinais clínicos relacionados (ASTRAUKAS ; CAMARGOS, 2013; DODI, 2015). 


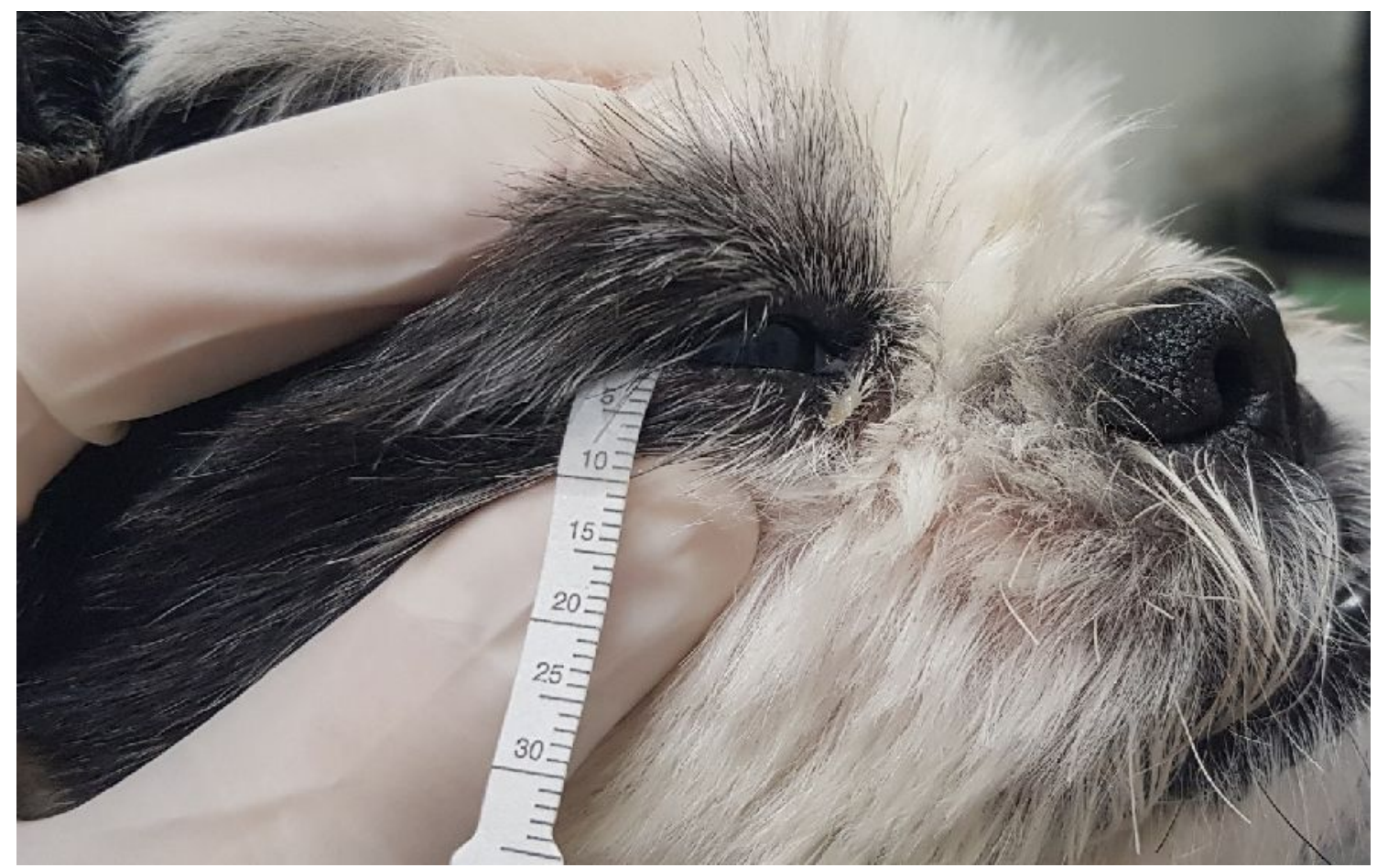

FIGURA 4 - Realização de teste lacrimal de Schirmer em cão. Fonte: Andréia Amaral

Para realização do método TLS-II é necessário a prévia realização de anestesia tópica, levando ao bloqueio do ramo aferente do reflexo prevenindo a secreção reflexa pelas glândulas lacrimal e terceira pálpebra, sendo possível a mensuração da produção basal e residual da lágrima (LIMA et al., 2014; JORGE, 2015). É considerado normal o valor entre 6 e $11 \mathrm{~mm} / \mathrm{min}$, sendo considerado positivos para CSS valores abaixo de 6 (JORGE, 2015).

O diagnóstico é estabelecido com base nos sinais clínicos típicos, na realização dos exames oftálmicos específicos com corantes vitais (fluoresceína e rosa bengala) e no Teste Lacrimal de Schirmer reduzido. No entanto, em cães que apresentam distúrbios nas células caliciformes conjuntivais ou das glândulas tarsais ou meibomianas, podem apresentar sinais clínicos de CCS, mas mantêm as medidas do TLS geralmente dentro do padrão de normalidade (COLITZ, 2008).

\section{Tratamento}

O tratamento medicamentoso da CCS inclui lacrimogênicos, lacrimomiméticos, mucolíticos e antibacterianos tópicos. Havendo evidências da etiologia autoimune a preferência pelo uso de Ciclosporina A tópica que é indicada como tratamento de eleição para estimular a produção lacrimal (ASTRAUSKAS ; CAMARGO, 2013).

Uma abordagem múltipla pode ser necessária para a terapia do olho seco, conservação e substituição da lágrima por meio de colírios anti-inflamatórios lacrimoestimulantes e lacrimomiméticos e, em alguns casos, procedimentos cirúrgicos podem ser necessários como transposição do ducto parotídeo, oclusão do ducto lacrimal ou transplante de glândula salivar podem ser necessários. Os imunomoduladores como ciclosporina e tacrolimo vem apresentando resultados promissores como terapia adjuvante no tratamento da CCS. Ambos atuam na redução da infiltração de linfócitos nas glândulas lacrimais e modulam a atividade celular por meio da supressão das células epiteliais através da inibição da enzima calcineurina (VOITENA et al., 2018). 
A Ciclosporina A é um efetivo imunossupressor, tem o efeito de aumentar a produção de lágrimas através de um mecanismo que inibe atividades de células $T$, citocinas inflamatórias, tanto na conjuntiva quanto na glândula lacrimal. Favorece o aumento da densidade das células caliciformes e diminui a apoptose de células epiteliais na conjuntiva (FULGÊNCIO et al., 2013).

A prescrição de ciclosporinaa $A$ tópica pode ser feita inicialmente a cada 12 horas em casos mais brandos, já nos casos mais graves pode ser administrava a cada 8 horas. Esse tratamento deve ser contínuo, e para que o aumento significativo da produção lacrimal seja observado, várias semanas de tratamento são necessárias. Dessa forma CsA de forma geral, reduz a conjuntivite mucopurulenta e promove cicatrização de úlceras corneanas, sendo uma utilização segura nesses casos (BIONDI et al., 2010).

Implantes oculares de liberação contínua foram desenvolvidos. Essa técnica tem por vantagem auxiliar no manejo com o animal, de modo que o tutor não necessite administrar o clírio a cada 8 a 12 horas todos os dias durante semanas, o que facilita o tratamento. Esses implantes permitem o fornecimento de níveis terapêuticos constantes do fármaco para os olhos. São úteis no tratamento de problemas oculares crônicos, como por exemplo em cães com ceratoconjuntivite superficial crônica.

Quanto aos tipos de implantes, podem ser adquiridos implantes sólidos, como por exemplo os de silicone, os biodegradáveis que são feitos de PGLA, quitosana, entreo outros, suspensões de micropartículas e géis injetáveis termossensíveis. É importante saber que os implantes intraesclerais podem ser colocados em qualquer segmento ocular, os de subconjuntiva/episcleral são utilizados para doenças do segmento anterior, e já os implantes intravítreos ou supracorroidais são usados para acometimentos no segmento posterior (BARACHETTI et al., 2014).

O tacrolimus possui efeitos similares ao da ciclosporina, além da imunossupressão local, causa supressão de apoptose das células lacrimais e possui uma ação anti-inflamatória. Os ácidos graxos essenciais como o ômega 3 e ômega 6 são agentes anti-inflamatórios naturais, dessa forma, o óleo de linhaça e óleo de oliva são frequentemente adicionados em colírios de tacrolimus, sendo que o óleo de linhaça possui uma ação anti-inflamatória mais eficiente (ZULIM et al., 2018).

A CCS neurogênica frequentemente responde ao uso de pilocarpina. Deverá ser utilizada a dose de 1 gota de pilocarpina a $2 \%$ para cada dez quilos de peso corporal, a cada oito horas, por um período que poderá variar de acordo com melhora clínica da causa primária. Geralmente os tratamentos duram por volta de seis meses (LEIVA; GIMÉNEZ, 2017; WILLIAMS, 2017).

Embora eficaz, o tratamento clínico não funciona em todos os casos, $\mathrm{O}$ tratamento cirúrgico é utilizado em casos de problemas oftálmicos crônicos, como por exemplo em cães com ceratoconjuntivite seca crônica e em casos refratários à terapia medicamentosa (BARACHETTI et al.; 2014). Nesses casos pode ser implantado um enxerto composto por mucosa labial e glândulas salivares subjacentes, esse tratamento tem como resultados: alívio dos sintomas do olho seco já nos primeiros dias, além da melhora subjetiva da acuidade visual.

O trabalho de Cherry et al. (2018) propôs o transplante de mucosa labial para o tratamento da ceratoconjuntivite seca canina. Eles avaliaram mucosas de cães eutanasiados com o propósito de encontrar glândulas salivares menores, as amostras foram obtidas por punção de $8 \mathrm{~mm}$ na mucosa labial rostral inferior na linha média, na mucosa labial rostral superior na linha média, mucosa labial superior 
próxima à comissura, na mucosa labial inferior próxima à comissura e na mucosa aproximadamente $1 \mathrm{~cm}$ caudal à comissura. Porém em nenhum dos locais selecionados foram encontradas células MSG ou outras células secretórias. Pôde ser concluído então que as glândulas salivares menores após o transplante de mucosa labial não são eficientes para o tratamento da ceratoconjuntivite seca.

A cirurgia de transposição do ducto parotídeo é um tratamento a ser considerado. O transplante de mucosa oral com glândulas salivares menores (MSG) tem sido descrito em pacientes humanos com olho seco grave. (CHERRY et al., 2018).

De acordo com Villatoro et al. (2014) um novo protocolo para o tratamento de CCS está sendo estudado. Sabe-se que a causa mais comum do olho seco é inflamatoria imunomediada. Os tratamentos clínicos por serem de uso longo e contínuo e muitas vezes tem uma resistência por meio dos tutores, além de desencadear efeitos colaterais que limitam o uso muito prolongado de certas drogas. Dessa forma, a utilização transplante de tecido adiposo como um novo tratamento para CCS é indicado por meio do autor. O tecido adiposo além de ser um tecido acessível e abundante para se obter essas células mesenquimais, possui células estromais mesenquimais (Ad-MSCs) que são células tronco com capacidade osteogênica, miogênica, secretora, e função imunomoduladora.

O autor ainda relata que reimplantações podem ser necessárias caso os efeitos terapêuticos não sejam suficientes após a primeira implantação, novas implantações podem ser feitas várias vezes sem provocar alteração celular imune. Em conclusão esse implante é um método seguro, relativamente simples de tratamento de ceratoconjuntivite seca, com uma melhora significativa na produção de lágrimas (VILLATORO et al., 2014).

\section{CONSIDERAÇÕES FINAIS}

A ceratoconjuntivite seca constitui uma condição frequente na clínica de cães. Para o diagnóstico devem ser considerados os fatores predisponentes, tóxicos, traumáticos, infecciosos, endócrinos, neurológicos dentre outros, apesar de a causa imunomediada ou idiopática ser a mais frequente. A escolha do tratamento deverá incluir a causa, a gravidade e os sinais clínicos, sendo que o tratamento medicamentoso é primordialmente indicado em relação às técnicas cirúrgicas. $O$ teste de Schirmer ainda constitui o teste padrão para o diagnóstico da CCS quantitativa. $O$ clínico deve se manter atualizado quanto às novas possibilidades no tratamento da CCS, de forma a ter métodos alternativos frente ao paciente com CCS irresponsiva aos tratamentos convencionais.

\section{REFERÊNCIAS}

ANGÉlICO, G. T.; RANZANI, J. J. T.; BRANDÃO.; S. A.; SHELLINI.; S. A. PADOVANI.; C. R. et al: Transplante de glândulas salivares menores no tratamento da ceratoconjuntivite seca em cães. Arquivo Brasileiro Medicina veterinária e zootécnica , v. 63, n. 5, p.1087-1092, 2011. Disponível em: <http://www.scielo.br/scielo.php?pid=S01029352011000500007\&script=sci_arttext>.

ASTRAUSKAS, J. P.; CAMARGOS, A. S: Ceratoconjuntivite seca em cães - revisão de literatura. Revista Científica Eletrônica de Medicina Veterinária, v.1, n. 20, p. 29-37, 2013. Disponível em: <http http://faef.revista.inf.br/imagens_arquivos/arquivos_destaque/ViCuy7uJTqJbJIH_201 3-6-21-16-1-7.pdf> 
BARACHETTI,L .;RAMPAZZO, A.; MORTELLARO, M, C.; SCEVOLA, S.; GILGER, C, B. Use of episcleral cyclosporine implants in dogs with keratoconjunctivitis sicca: pilot study. Veterinary Ophtalmology, v-18, p, 234-241. 2014. 10.1111/vop.12173. Disponível em: $<$ https://www.vetweb.com.br/newgaleria/gallery/admin/rest/arquivos_agenda/Barache tti_et_al-2015-Veterinary_Ophthalmology.pdf>

BIONDI, F. Ceratoconjuntivite seca. Revista Científica de Medicina Veterinária Pequenos Animais e Animais de Estimação, v. 8, p. 93-98, 2010. Disponível em: <http://medvep.com.br/wp-content/uploads/2017/01/Artigo057.pdf>

BIRCHARD, S. J.; SHERDING, R. G. Manual Saunders - Clínica de Pequenos Animais. 3ed. São Paulo: ROCA, p, 2008-2048.

CASTILLO, B, M, J.; How to promote and preserve eyelid health. Clinical Ophtalmology published online, 2012. Disponível em: <https://www.ncbi.nlm.nih.gov/pmc/articles/PMC3484726/>

CHERRY, L, R.; SMITH, D, J.; SHLOMO, B, G. Canine oral mucosa evaluation as a potential autograft tissue for the treatment of unresponsive keratoconjunctivitis sicca. Veterinary Ophtalmology. v.21, p. 48-51, 2017. Disponível em: <https://onlinelibrary.wiley.com/doi/abs/10.1111/vop.12477>

COLITZ, C. M. H. Doenças do sistema lacrimal. In__: BIRCHARD, S. J.SHERDING, R. G. Manual Saunders: clínica de pequenos animais. 3ed. São Paulo: Roca, 2008, cap. 139, p. 1416-1421.

DESTEFANIS, S.; GIRETTO, D.; MUSCOLO, M, C.; DI CERBO, A.; GUIDETTI, G.; et al: Clinical evaluation of a nutraceutical diet as an adjuvant to pharmacological treatment in dogs affected by Keratoconjunctivitis sicca. BMC Veterinary Research. v.12, p. 214, 2016 Disponível em: <https://www.ncbi.nlm.nih.gov/pubmed/27658509>

DODI, L, P. Immune-mediated Keratoconjuntivitis sicca in dogs: current perspectives on management. Departamento f Veterinary Medicine Sciences, v. 2015, p. 341347, 2015.2 Disponível em: <https://www.researchgate.net/profile/Pier_Luigi_Dodi/publication/283682435_Immun e-

mediated_keratoconjunctivitis_sicca_in_dogs_current_perspectives_on_managemen t/links/56937e0008aec14fa55e8c24/Immune-mediated-keratoconjunctivitis-sicca-indogs-current-perspectives-on-management.pdf>

FERRAZ, M. F. S.; SOUZA, M. S. B. Análise da produção de lágrima em pacientes com alopecia periocular: estudo retrospectivo entre os anos de 2008 e 2012 no Hospital Veterinário da Universidade Estadual de Londrina - Ciências Agrárias. v. 35 , n.4, suplemento, p. 2443-2452, 2014. DOI: 10.5433/16790359.2014v35n4Suplp2443

FULGÊNCIO, O, G.; SALIBA, B, J.; FIALHO, L, S.; JÚNIOR, C, S, A. Sistema de liberação contendo ciclosporina para o tratamento de ceratoconjuntivite seca: estudo preliminar. Revista Brasileira de Oftalmololgia. v. 72, n. 4, p. 232, 2013. Disponível em:<https://www.researchgate.net/profile/Juliana_Saliba/publication/262662670_Cycl 
osporineloaded_delivery_system_for_the_treatment_of_keratoconjunctivitis_sicca_A pilot_study/links/545bf7630cf249070a7a8508/Cyclosporine-loaded-delivery-systemfor-the-treatment-of-keratoconjunctivitis-sicca-A-pilot-study.pdf>

GELATT, K. N. Veterinary Ophthalmology. USA; John Wiley \& Sons, 2013. Cap. 16, V. 2, p. 912-932.

HERRERA, D. H. Enfermidades palpebrais. In: .Oftalmologia clínica em animais de companhia. São Paulo: Editora MedVet, p. 89-100, 2008a.

J.N. VOITENA' J, N.; CUNHA, O.; FUKUSHIMA, B, F, CARVALHO, F, G.; RAMOS, $S$, C, L.; et al: Efficacy of cyclosporine and tacrolimus drops in the treatment of keratoconjunctivitis sicca in dogs. Arquivo Brasileiro de Medicina Veterinária e Zootecnia. v.70, 2018. Disponível em: <http://dx.doi.org/10.1590/1678-4162-9849>

JORGE, T, A.; HONSHO, S, C.; PEREIRA, F, L.; DIAS, G, G, G,; DIAS, G, G, F. Diferentes respostas ao tratamento clínico na ceratoconjuntivite seca em cães. Enciclopédia Biosfera, v.11 n.21; p. 764, 2015. Disponível em: <file:///C:/Users/Jo\%C3\%A3o/Downloads/diferentes\%20respostas.pdf >

LAUS, J. L. Oftalmologia Clínica e Cirúrgica em cães e gatos. São Paulo: Rocca: 2009. Pg. $77-82$

LEIVA, M.; GIMÉNEZ, T. R. Diseases of the lacrimal system. In: MAGGS, D.; MILLER, P.; OFRI, R. Slatter's Fundamental's of Veterinary Ophthalmology. 6 ed. St Louis: Elsevier, 2018. p.186-212.

LIMA, F. B.; ORIÁ, A. P.; MENEZES, I. D. S.; RAPOSO, A. C. S.; GOMES JÚNIOR, D. C. Citologia esfoliativa em cães com ceratoconjuntivite seca. Enciclopédia Biosfera. v. 10, n. 18, p. 903-910, 2014. Disponível em: <http://www.conhecer.org.br/enciclop/2014a/AGRARIAS/Citologia\%20Esfoliativa.pdf $>$

MOTTA, D. A.; YAMASAKI, L.; SANCHES, O. C.; GIUFFRIDA, R.; CÂNDIDO, E. R.; et al:Comparação entre dois protocolos de tratamento de ceratoconjuntivite seca experimentalmente induzida em coelhos. Arquivo Brasileiro de Medicina Veterinária e Zootecnia. v. 66, n. 1, p. 47-54, 2014. Disponível em: <http://www.scielo.br/scielo.php?pid=S0102-

$09352014000100008 \&$ script=sci_arttext\&tlng=pt $>$

ORIÁ, P, A.; FURTADO, A, M.; JUNIOR, S, S, E.; PINNA, H, M. Ceratoconjuntivite Seca em cães Pubvet, V. 4, 2010. Disponível em: <http://www.pubvet.com.br/uploads/6126840155d2c3cea6e2329e0c8706d7.pdf>

SGRIRNOLI, R, M.; YAMASAKI, L.; SANCHES, C, O.; VALLE, D, F, H.; ZULIM, C, F, L.; et al: Comparison of Topical $0.03 \%$ Tacrolimus in Almond and Linseed Oil to Treat Experimentally Induced Keratoconjunctivitis Sicca in Rabbits. Ophtalmic Phatologhy. $\quad$ v. 2, 2013. 2 Disponível em: <https://www.researchgate.net/profile/Silvia_Franco_Andrade/publication/262172453 _Comparison_of_topical_003_tacrolimus_in_almond_and_linseed_oil_to_treat_expe 
rimentally_induced_keratoconjunctivitis_sicca_in_rabbits/links/54cff88e0cf29ca81100 bb5f.pdf>

SLATTER, D. Fundamentos de Oftalmologia Veterinária. São Paulo: Rocca: 2005. Cap. 11, p. 283-338.

TILLEY, L. P.; SMITH JUNIOR, F. W. K: Consulta veterinária em 5 minutos: espécies canina e felina. 3.ed. Barueri: MANOLE, 2008. 1550p.

VILLATORO, J, A.; FERNÁNDEZ, V.; CLAROS, S.; RICO-LLANOS, A, G.; BECERRA, J.; et al: Use of Adipose-Derived Mesenchymal Stem Cells in Keratoconjunctivitis Sicca in a Canine Model. BioMed Research International. V 10, 2015. Disponível em: < https://www.hindawi.com/journals/bmri/2015/527926/abs/> http://dx.doi.org/10.1155/2015/527926>

WILLIAMS, D. L. Canine Keratoconjunctivitis Sicca: Current concepts ind Diagnosis and Treatment. Journal of Clinical Ophthalmolog and Optometry. v.2, n.1. p.1-7, 2017. Disponível em: <http://www.scienceinquest.com/open-access/pdf/jcoo/caninekeratoconjunctivitis-sicca-current-concepts-in-diagnosis-and-treatment.pdf>.

ZULIM, C, F, L.; NAI, A, G.; GIUFFRIDA, R.; PEREIRA, G, S, C.; BENGUELLA, H.; et al: Comparison of the efficacy of $0.03 \%$ tacrolimus eye drops diluted in olive oil and linseed oil for the treatment of keratoconjunctivitis sicca in dog. Arquivo Brasileiro de Oftalmologia. v. 81, p. 293-301, 2018. Disponível em: <http://dx.doi.org/10.5935/0004-2749.20180059>. 